\title{
Proteinuria in Hypertensive Nephropathy: A Review
}

\author{
Rishi Sharma', Surineni Kamalakar¹, Ellen McCarthy², Timothy A. Fields², Kamal Gupta², \\ Rajat Barua1, Virginia Savin1 \\ ${ }^{1}$ Kansas City VA Medical Center, 4801 Linwood Boulevard, Kansas City, USA \\ ${ }^{2}$ University of Kansas Medical Center, 3901 Rainbow Boulevard, Kansas City, USA \\ Email: sharmarishi77@gmail.com
}

Received 17 April 2014; revised 16 May 2014; accepted 10 June 2014

Copyright (C) 2014 by authors and Scientific Research Publishing Inc.

This work is licensed under the Creative Commons Attribution International License (CC BY). http://creativecommons.org/licenses/by/4.0/

(c) (i) Open Access

\begin{abstract}
Hypertension defined as a systolic blood pressure of $\geq 140$ and a diastolic blood pressure $\geq 90$ is an extremely prevalent condition; and it is responsible for significant mortality and morbidity. NHANES data from $2005-2006$ found that nearly $30 \%$ of adult US population has HTN; and nearly $8 \%$ of the population has undiagnosed HTN. HBP mortality in 2008 was 61,005 . Any mentioned mortality in 2008 was 347,689 (NHLBI tabulation of NCHS mortality data). More than $20 \%$ of patients with systemic hypertension have chronic renal insufficiency (NHANES). Hypertensive nephropathy is a leading cause of end-stage renal disease (ESRD) requiring dialysis or transplantation or leading to death. The incidence of hypertension is high but only a subset of hypertensive patients progress to frank renal failure. A subset of hypertensive patients develop proteinuria during the course of disease and manifest nephrotic syndrome. This syndrome includes marked proteinuria, edema, and low serum albumin. Neither the incidence nor the clinical significance of proteinuria in hypertension without diabetes is known. Progression to chronic renal failure in some patients is preceded by proteinuria as indicated on "dip-stick" analyses of random urine samples. It appears that proteinuria is likely to increase both prior to and during evident loss of glomerular filtration, but this clinical observation has never been formally confirmed. There is a need for large studies to answer these questions. We also need to focus on the roles that genetic and environmental factors play in development and progression of renal disease in the setting of hypertension and proteinuria.
\end{abstract}

\section{Keywords}

Hypertension, Nephropathy, Proteinuria, ESRD, Genetics 


\section{Introduction}

Nearly 30\% of adults in the United States have known hypertension (HTN), while an additional 8\% have undiagnosed HTN [1]. Mortality attributed to HTN in 2008 was over 61,000 [2]. HTN affects many organ systems and causes end-organ damage to heart, blood vessels, brain and kidney. More than $20 \%$ of patients with systemic HTN have hypertensive nephropathy [3]. Fortunately, only a minority of these patients progress to advanced chronic kidney disease (CKD) or ESRD [4]. Hypertensive renal disease is the second most common cause of chronic renal failure requiring dialysis or renal transplantation in the US (end-stage renal disease, ESRD) and accounted for about 158,000 patients entering the USRDS system during 2006-2012 (28\% of incident patients) (USRDS report-2012) [5]. The mortality of these patients in the first year after initiating treatment for ESRD was about 24\%. Thus, the renal impact of HTN is second only to that of Type 2 diabetes mellitus, the renal diagnosis for about $45 \%$ of incident ESRD patients.

A subset of hypertensive patients developed proteinuria during the course of disease and manifested nephrotic syndrome, characterized by marked proteinuria, edema, and low serum albumin [6]. The relationship between proteinuria, BP control, use of specific medications and renal outcomes is not clearly defined, and the aim of this review is to highlight some recent findings related to hypertension, proteinuria and progression of renal disease.

\section{Pathological Changes in Hypertensive Nephropathy}

The renal changes in HTN are often classified as benign versus malignant nephrosclerosis. Benign nephrosclerosis is a gradual and prolonged process and shows the characteristic histologic changes described below. It is far from benign, as it results in progressive renal injury in some patients [7]. Malignant nephrosclerosis occurs in presence of malignant HTN (DBP > 130 and accelerated end-organ damage) and progresses at an alarming rate and, if untreated, may lead to death from stroke, heart attack or renal failure within months [8].

Vascular changes in benign HTN are evident and depend on the vessel size. Larger arteries show fibrous intimal thickening, with duplication of the elastic layer (fibroelastosis), as well as medial thickening. Small arterioles often exhibit hyaline arteriolosclerosis, characterized by subendothelial deposition of homogeneous eosinophilic material. Glomeruli are also damaged and exhibit two predominant patterns of injury: collapse and solidification. The collapsed glomerulus in benign HTN shows wrinkling and thickening of capillary basement membranes, collapse of capillary lumina, and ultimately retraction of the tuft to the vascular pole. This is accompanied by deposition of collagenous material within Bowman's space. The solidified glomerulus shows segmental or global sclerosis, accompanied by an increase in mesangial matrix [9]. Renal tubules and interstitium undergo scarring and renal cortex becomes thin and irregular. The degree of interstitial scarring is correlated with decreased glomerular filtration and progression to renal failure [10]. Examples of renal histology in a 54-year-old African American patient with longstanding HTN, nephrotic syndrome and CKD due to HTN are shown in Figure 1.

Vessels in malignant HTN often show features of thrombotic microangiopathy (i.e., mucoid intimal thickening, mural fibrinoid necrosis, red blood cell fragmentation and extravasation, and thrombosis). These acute vascular changes superimposed upon the changes in benign HTN can create an exaggerated layered appearance (onion skinning) of the vessel. Glomeruli may exhibit a variety of changes, including capillary loop thickening with double contours, segmental fibrinoid necrosis, occlusive endothelial swelling, capillary thrombi, and attenuation of mesangial matrix (mesangiolysis). Characteristic glomerular global sclerosis, arteriolar nephrosclerosis and interstitial fibrosis may be absent in malignant hypertension.

Patients with renal insufficiency in the setting of HTN and absence of other known systemic disease are often labeled as having hypertensive nephrosclerosis without supportive renal biopsy findings. Primary renal disease in patients with hypertensive nephropathy may be more common than is currently appreciated. This potentialmisdiagnosis may impact the epidemiology, evaluation and treatment of patients with ESRD. Thus, caution must be used in making the diagnosis of hypertensive nephropathy in the absence of confirmatory pathology [11].

\section{Proteinuria in Hypertensive Nephropathy}

Microalbuminuria, macroalbuminuria and nephrotic syndrome may each occur in patients with hypertensive nephropathy. Microalbuminuria is a non-specific finding in patients with vascular disease and is associated with increased risk of cardiovascular events. The majority of patients with benign nephrosclerosis have proteinuria in 


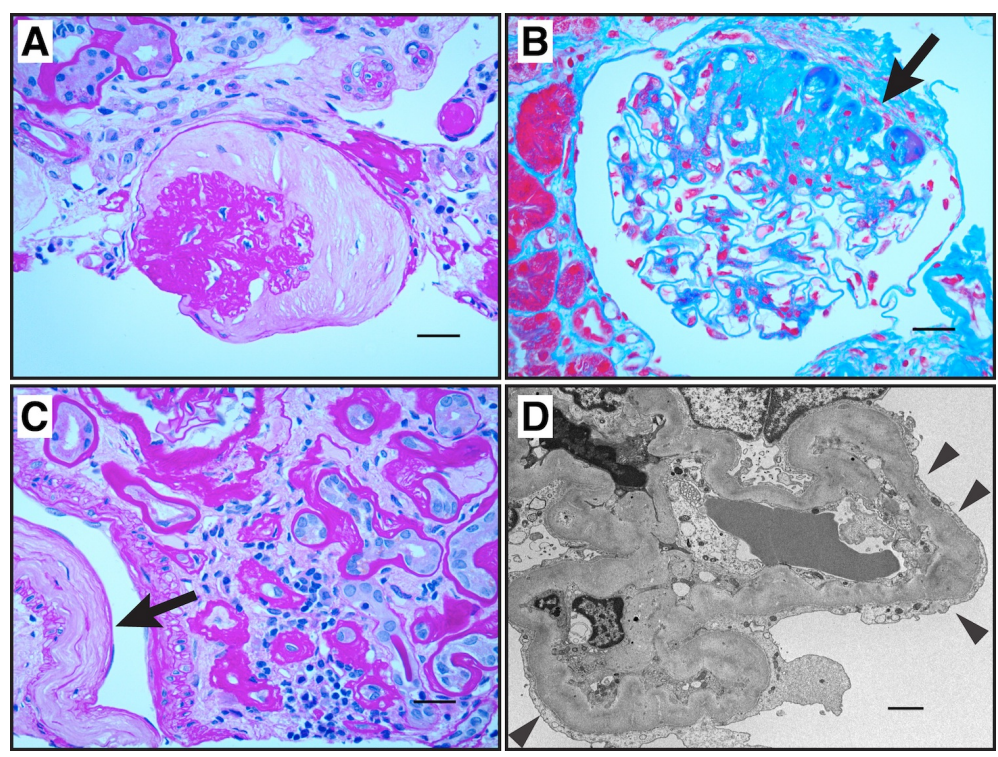

Figure 1. Renal biopsy from 54-year-old African American patient with history of multiple hospitalizations for hypertensive urgency. (A) Light micrograph showing hypertensive glomerular lesion: global glomerular collapse and filling of Bowman's space with a lightly staining collagenous material (PAS stain, scale bar $=25 \mu \mathrm{m}$ ). (B) Light micrograph of glomerulus showing secondary segmental sclerosis (arrow; Masson trichrome stain, scale bar $=25$ $\mu \mathrm{m})$. (C) Light micrograph showing interstitial fibrosis, tubular atrophy with thickened tubular basement membranes, and fibrous intimal thickening of a small artery (arrow; PAS stain, scale bar $=25 \mu \mathrm{m}$ ). (D) Transmission electron micrograph showing wrinkling and thickening of glomerular basement membrane. There is extensive foot process effacement in this segment (arrowheads; scale bar $=2 \mu \mathrm{m}$ ). Immunofluorescence studies for IgG, IgA, and complement were negative; there was minimal staining for IgM in areas of focal sclerosis (not shown). At the time of biopsy, eGFR was $25 \mathrm{ml} / \mathrm{min} \cdot 1.73$ $\mathrm{m}^{2}$ and urine protein/creatinine ratio was $6.5 \mathrm{~g} / \mathrm{g}$. Rate of decline in eGFR had been about $10 \mathrm{ml} / \mathrm{min} \cdot 1.73 \mathrm{~m}^{2}$ per year for the last 5 years.

the range of 0.5 to $1 \mathrm{~g} / 24$ hours. More pronounced proteinuria also occurs in hypertensive renal disease. In one study of 145 patients with hypertensive nephrosclerosis who underwent renal biopsy between 1960 and 1982, only 18 (12\%) had nephrotic syndrome [12]. Six of these patients had malignant HTN, while the remaining 12 had nephrotic range proteinuria but only benign nephrosclerosis. Patients with both benign and malignant nephrosclerosis exhibited arteriosclerosis, medial thickening, and duplication of intima. In addition, patients with malignant nephrosclerosis had arteriolar lesions with fibrinoid necrosis and fragmentation of the elastic lamina. Authors suggested that hypertensive nephropathy should be included in the differential diagnosis of nephrotic range proteinuria in patients with azotemia and poorly controlled HTN and pointed out that benign HTN may lead to nephrotic syndrome [12].

In a 2002 a study was conducted to determine the frequency of nephrotic range proteinuria in patients with hypertensive nephrosclerosis. Researchers reviewed biopsy reports on 237 consecutive cases of nephrotic syndrome in African American patients from 1993 to 2000. Patients with diabetes mellitus, systemic lupus erythematous, hepatitis, human immunodeficiency virus (HIV) or chronic use of non-steroidal anti-inflammatory agents were excluded. Patients whose biopsy specimens showed glomerular eosinophilic hyalinosis, positive immunofluorescence staining, or dense deposits on electron microscopy were also excluded. Of the 237 patients, 13 met the histologic criteria for hypertensive nephrosclerosis. Thus, more than $5 \%$ of cases of nephrotic range proteinuria were attributed solely to hypertensive nephrosclerosis [13]. An examination of a primarily Caucasian hypertensive population confirmed the presence of marked proteinuria in some patients and showed that lower serum albumin, albuminuria, and higher serum creatinine were correlated with rapid loss of renal function. The presence of elevated creatinine or microalbuminuria has been suggested as a screen for identifying people at 
high risk of cardiovascular and renal complications [14].

Only a faction (20\%) of patients with HTN develops hypertensive nephropathy. Both genetic and environmental factors affect risk as illustrated in Figure 2.

\section{Progression of Renal Disease in Hypertension in African Americans}

Hypertension is more prevalent, of earlier onset and more severe in African Americans (AA) and AAs have an increased risk of ESRD from hypertensive nephropathy [15]. The difference in pattern of disease has led to focus on mechanisms or risk factors unique to AAs. The AASK trial was intended to correct prior under-representation of AA in studies of the cardiovascular and renal effects of HTN and test the postulate that a lower BP goal might be effective in slowing the progression of kidney disease in AA [16]. This randomized, double blind, multicenter study was designed to determine the effects of BP control and the class of antihypertensive drug on kidney function in AA with hypertensive nephropathy [17]. A total of 1094 AA patients age 18 - 70 years, with GFR 20 - $65 \mathrm{~mL} / \mathrm{min}$ per $1.73 \mathrm{~m}^{2}$, were enrolled. They were randomly assigned to 2 levels of BP control, defined by mean arterial pressure, and to initial treatment with a beta-blocker, an angiotensin converting enzyme inhibitor (ACEI) or a dihydropyridine calcium channel blocker. Additional diuretic, sympathetic and vasodilatory antihypertensive agents were added to achieve BP targets. The primary outcome was the rate of decline of GFR. Secondary outcomes were reduction of GFR by 50\% or more from the baseline, ESRD and all-cause mortality. Surprisingly, there was no significant difference between BP groups in rate of GFR decline, composite end points or cardiovascular events. However, the GFR did decline more rapidly in patients with urine proteincreatinine ratio of more than 0.22 and in those in the calcium channel blocker group vs. the ACEI group. Baseline proteinuria predicted rapid progression of renal disease, and this effect was exaggerated in patients assigned to the low BP control target. Treatment with ACEI slowed progression of renal disease in all groups, regardless of proteinuria. A subsequent cohort study with this same AASK patient population was performed, setting a BP goal of 130/80. Patients were followed for 9 - 12 years, and the data suggested that lower BP targets may slow progression selectively in those with proteinuria at baseline [18].

\section{Genetic Analyses in Hypertension}

Genetics plays an important role in many diseases, including HTN-related CKD. The high rate of progressive renal disease in African American patients with HTN and the strong predictive value of family history of ESRD are consistent with a genetic contribution to risk of renal failure. Mapping by admixture disequilibrium identified a region on chromosome 22 that contains 2 candidate genes, apolipoprotein-L1 (APOL1) and non-muscle myosin heavy chain type 2 (MYH9). Both MYH9 and APOL1 are expressed in glomerular podocytes and could predispose these cells to injury and accelerated glomerular sclerosis. Initial studies showed a strong association

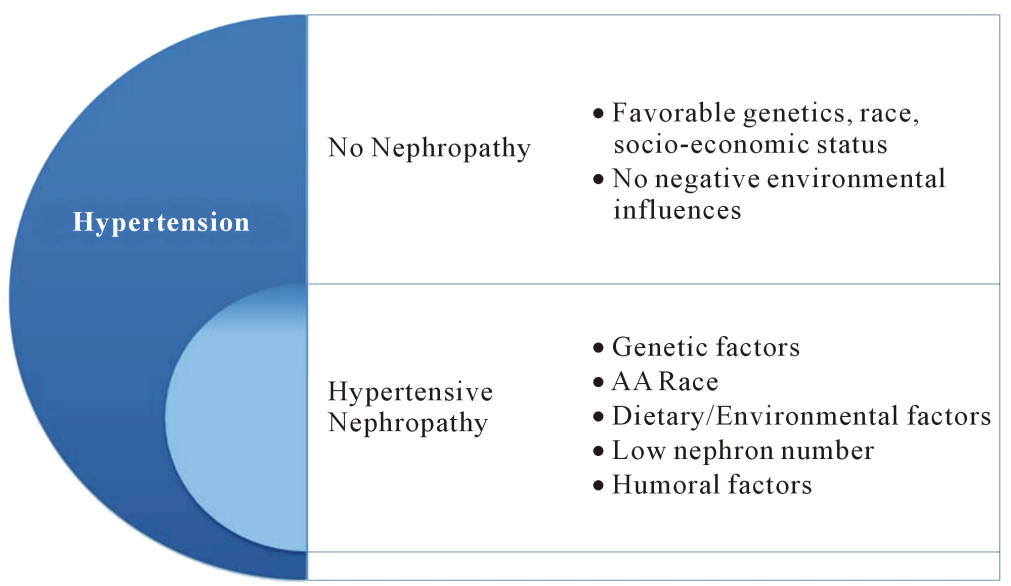

Figure 2. Proposed mechanisms for proteinuria and progression of renal injury in hypertensive nephropathy. Only a faction (20\%) of patients with HTN develop hypertensive nephropathy. Both genetic and environmental factors affect risk. 
of MYH9 with FSGS and HIV-associated nephropathy [19]. However, the APOL1 gene is more strongly associated with FSGS and HTN-related CKD in AA patients [20]. A recent case control study of AASK patients examined the association between APOL1 and MYH9 and HTN-related kidney disease [21]. APOL1 risk variants were associated with kidney disease (odds ratio of 2.57). This association was stronger in patients with more advanced disease and was independent of medication classes and blood pressure targets to which patients had been assigned. MYH9 risk alleles seem to play a more important role in individuals of European ancestry and are associated with increased risk of both non-diabetic chronic kidney disease (CKD) and diabetic ESRD [22]. APOL1 genotypes were not associated with renal risk in patients of European ancestry.

Genome-wide studies continue to identify new genes that are more common in HTN than in others. Most recently 5 loci (AGT, CYP11B1, NR3C2, SCNN1G, and WNK1) with higher association at evolutionary conserved sites were identified. Both rare and common variants at these genes affect BP in the general population with modest effects [23]. Larger sample sizes will be required to determine the individual contributions of these and other genes. Additional investigations of genetics may provide clinically useful insights into the clinical and histologic differences in hypertensive nephrosclerosis in AA and European Americans.

RAS plays a critical role in hypertension. Several studies have focused on polymorphisms in genes coding for RAS system. Among these, polymorphisms for ACE and AT1R gene are most researched. Despite many studies no association has been found between polymorphism of ACE/AT1R and HTN or ESRD. Thus, at this point there is no clinical indication for basing therapeutic strategies on the genotype of ACE/AT1R genes [24].

\section{Potential Role of Diet, Nutrition, and Lifestyle}

CKD and HTN are each prevalent worldwide, but incidence may vary among countries. For example, the incidence of early CKD (Stage 1 and 2) among Caucasians in the US and Norway are similar, while the rates of ESRD are lower in Norway [25]. This and other observational studies raise the question of the role of diet, lifestyle and patterns of medical practice in progression of renal disease. Notable differences include the higher rate of diabetes mellitus and obesity and later referral to care by nephrology in the US.

Diet may play an important role in the cause and progression of various diseases including HTN, CKD, and cardiovascular diseases. The prevalence of HTN in the USA has significantly increased during the past 75 years. In 1939, 11\% - 13\% of adult 11,000 residents of Chicago had HTN; the prevalence of HTN had increased to 31\% by 2004 [26]. Changes in dietary habits during this period include increased use of fat, sugar and salt. Each of these dietary components can contribute to the pathogenesis of HTN, CAD and CKD. In addition, uric acid derived from fructose metabolism appears to contribute to vascular endothelial dysfunction and lead to increased atherogenesis, HTN, metabolic syndrome, and cardiovascular mortality [27]. Salt itself contributes to HTN in many animal models and is postulated to be a leading determinant of HTN in humans [28]. Acid generation, reflected by urinary acid excretion and low normal plasma bicarbonate levels, may also affect progression of renal disease in HTN and may be treated by addition of fruits and vegetables or oral sodium bicarbonate to the daily diet [29]. An additional effect of diet may occur through influences of maternal obesity or diabetes mellitus or because of prematurity or intrauterine growth delay. Each of these may be associated with low nephron number, which leads to increased hemodynamic and humoral stress, podocyte damage and glomerular scarring [30]. The precise contribution of diet in HTN and hypertensive nephropathy remains to be determined.

\section{Novel Interventions in the Treatment of Hypertension}

Current interventions have limited efficacy in preventing progression in hypertensive renal disease. Loss of renal function may continue without apparent benefit from control of BP or the use of potentially protective agents, including those that target the renin-angiotensin-aldosterone system (RAAS). New drug targets are being identified and innovative approaches are being developed to treat HTN and associated renal disease.

Endothelin is a peptide that has potent vasoconstrictor activity and can elicit other biological effects, including stimulation of cell proliferation. It is biologically active in HTN, renal failure, CAD, heart failure and idiopathic cardiomyopathy. Endothelin receptor A/B blocker (Bosentan) initially showed promise in treating conditions in which endothelin plays a role [31]. However, this promise has not led to efficient and safe clinical use. Additional receptor-specific endothelin receptor inhibitors are currently being tested.

ACEIs, angiotensin receptor blockers, aldosterone inhibitors and renin inhibitors have each been widely used in treating HTN. Immunization to the elements of the RAAS system is a novel strategy for treatment of HTN. A 
vaccine that induces neutralizing antibodies to angiotensin-I (PMD3117) has been developed and tested in phase I/II trials. PMD3117 showed safety and immunogenicity in humans, however, there was insufficient antibody response to effectively reduce HTN [32]. A second vaccine (CYT006-AngQb-a) that is effective against angiotensin-II has been tested in patients with mild to moderate HTN. The vaccine was safe, well tolerated and effective [33]. Since angiotensin II has pro-fibrotic activity in both heart and kidney, it is possible that the vaccine could prevent these complications of long-standing HTN. Further testing is required to determine long-term risks and benefits of these and comparable vaccines before they can be used in the general population.

Surgical sympathectomy has been employed in the treatment of HTN, but its invasive nature and resulting autonomic insufficiency has limited its clinical use. Two new examples of interventions targeting sympathetic responses are implantation of carotid sinus nerve stimulating electrodes [34] and catheter-based radio frequency ablation of renal afferent and efferent nerves [35]. While these approaches each lower BP, they have not yet gained widespread use because their potential for decreasing proteinuria and slowing progression of renal disease, as well as their long-term risks, are not known. Moreover, in a recent study, renal denervation therapy had no beneficial effect in reducing BP in people with resistant hypertension [36].

The efficacy of early identification of patients at high risk for progression of CKD, early referral to subspecialty care, and specific treatment practices in HTN treatment are not fully understood. Use of computerized decision support (CDS) has been thought to improve care in other medical conditions, but a recent study of CDS in HTN treatment showed improved drug prescribing but no change in health care disparities or overall control of HTN [37].

\section{Conclusion}

Hypertensive kidney disease is a complex, multifactorial disease process and genetics, race, economic status, diet \& lifestyle, and the timing of diagnosis and treatment affects its natural history. Current therapies have limited efficacy in arresting progression of kidney disease in HTN. We propose that proteinuria may be early sign of significant renal injury in HTN, that progressive increase in proteinuria may be detected before there is a decrease in GFR, and that proteinuria may be a predictor of future decline in renal function and ESRD. If this is the case, proteinuria can be used to identify patients at high risk of renal failure. These patients can then receive more intensive empiric therapy to control BP and other known risk factors. In addition, they may prove to be an ideal population for the study of new and potentially effective therapies.

\section{Acknowledgements}

This material is the result of work supported with resources and the use of facilities at the Kansas City VA Medical Center, Kansas City, Missouri.

\section{References}

[1] Ostchega, Y., et al. (2008) Hypertension Awareness, Treatment, and Control--Continued Disparities in Adults: United States, 2005-2006. NCHS Data Brief, 3, 1-8.

[2] Centers for Disease Control and Prevention (2012) Vital Statistics Public Use Data Files-2008 Mortality Multiple Cause Files. http://www.cdc.gov/nchs/data_access/Vitalstatsonline.htm-Mortality_

[3] Rutkowski, B., Tylicki, L. and Debska-Slizien, A. (2003) Pathogenetic and Epidemiological Aspects of Hypertensive Nephropathy. Polskie Archiwum Medycyn Wewnetrznej, 110, 1167-1171.

[4] US Renal Data System (2011) USRDS. Annual Data Report: Atlas of Chronic Kidney Disease and End-Stage Renal Disease in the United States. National Institutes of Health, National Institute of Diabetes and Digestive and Kidney Diseases, Bethesda.

[5] Udani, S., Lazich, I. and Bakris, G.L. (2011) Epidemiology of Hypertensive Kidney Disease. Natural Review of Nephrology, 7, 11-21. http://dx.doi.org/10.1038/nrneph.2010.154

[6] Schmieder, R.E. (2010) End Organ Damage in Hypertension. Dtsch Arztebl International, 107, 866-873.

[7] Harvey, J.M., et al. (1992) Renal Biopsy Findings in Hypertensive Patients with Proteinuria. Lancet, 340, $1435-1436$. http://dx.doi.org/10.1016/0140-6736(92)92624-O

[8] D’Agati, V.D. and Mengel, M. (2013) The Rise of Renal Pathology in Nephrology: Structure Illuminates Function. American Journal of Kidney Disease, 61, 1016-1025. http://dx.doi.org/10.1053/j.ajkd.2012.12.019 
[9] Dasgupta, I., et al. (2007) “Benign” Hypertensive Nephrosclerosis. QJM, 100, 113-119. http://dx.doi.org/10.1093/qjmed/hcl139

[10] Nonaka, K., et al. (2013) Clinical and Pathological Evaluation of Hypertensive Emergency-Related Nephropathy. Internal Medicine, 52, 45-53.

[11] Freedman, B.I., et al. (1994) Renal Biopsy Findings in Presumed Hypertensive Nephrosclerosis. American Journal of Nephrology, 14, 90-94. http://dx.doi.org/10.1159/000168695

[12] Mujais, S.K., et al. (1985) Marked Proteinuria in Hypertensive Nephrosclerosis. American Journal of Nephrology, 5, 190-195. http://dx.doi.org/10.1159/000166931

[13] Obialo, C.I., Hewan-Lowe, K. and Fulong, B. (2002) Nephrotic Proteinuria as a Result of Essential Hypertension. Kidney Blood Press Research, 25, 250-254. http://dx.doi.org/10.1159/000066345

[14] Viazzi, F., Leoncini, G. and Pontremoli, R. (2013) Global Cardiovascular Risk Assessment in the Management of Primary Hypertension: The Role of the Kidney. International Journal of Hypertens, 2013, 542-646.

[15] Rahman, M., Douglas, J.G. and Wright Jr., J.T. (1997) Pathophysiology and Treatment Implications of Hypertension in the African-American Population. Endocrinology and Metabolism Clinics of North America, 26, 125-144. http://dx.doi.org/10.1016/S0889-8529(05)70237-1

[16] Striker, G.E. (1992) Current KUH Notations. American Journal of Kidney Diseases, 20, 673. http://dx.doi.org/10.1016/S0272-6386(12)70239-1

[17] Wright Jr., J.T., Bakris, G., Greene, T., Agodoa, L.Y., Appel, L.J., Charleston, J., et al. (2002) Effect of Blood Pressure Lowering and Antihypertensive Drug Class on Progression of Hypertensive Kidney Disease: Results from the AASK Trial. JAMA, 288, 2421-2431. http://dx.doi.org/10.1001/jama.288.19.2421

[18] Appel, L.J., Wright Jr., J.T., Greene, T., Agodoa, L.Y., Astor, B.C., Bakris, G.L., et al. (2010) Intensive Blood-Pressure Control in Hypertensive Chronic Kidney Disease. The New England Journal of Medicine, 363, 918-929. http://dx.doi.org/10.1056/nejmoa0910975

[19] Kopp, J.B., Smith, M.W., Nelson, G.W., Johnson, R.C., Freedman, B.I., Bowden, D.W., et al. (2008) MYH9 Is a Major-Effect Risk Gene for Focal Segmental Glomerulosclerosis. Nature Genetics, 40, 1175-1184. http://dx.doi.org/10.1038/ng.226

[20] Genovese, G., Friedman, D.J., Ross, M.D., Lecordier, L., Uzureau, P., Freedman, B.I., et al. (2010) Association of Trypanolytic ApoL1 Variants with Kidney Disease in African Americans. Science, 329, 841-845. http://dx.doi.org/10.1126/science.1193032

[21] Cooke, J.N., Bostrom, M.A., Hicks, P.J., Ng, M.C., Hellwege, J.N., Comeau, M.E., et al. (2012) Polymorphisms in MYH9 Are Associated with Diabetic Nephropathy in European Americans. Nephrology Dialysis Transplantation, 27, 1505-1511. http://dx.doi.org/10.1093/ndt/gfr522

[22] Lipkowitz, M.S., Freedman, B.I., Langefeld, C.D., Comeau, M.E., Bowden, D.W., Kao, W.H., et al. (2013) Apolipoprotein 11 Gene Variants Associate with Hypertension-Attributed Nephropathy and the Rate of Kidney Function Decline in African Americans. Kidney International, 83, 114-120. http://dx.doi.org/10.1038/ki.2012.263

[23] Nguyen, K.D., Pihur, V., Ganesh, S.K., Rakha, A., Cooper, R.S., Hunt, S.C., et al. (2013) Effects of Rare and Common Blood Pressure Gene Variants on Essential Hypertension: Results from the Family Blood Pressure Program, CLUE, and Atherosclerosis Risk in Communities Studies. Circulation Research, 112, 318-326. http://dx.doi.org/10.1161/CIRCRESAHA.112.276725

[24] Mao, S. and Huang, S. (2013) Lack of Association of Angiotensin II Type 1 Receptor A1166C Gene Polymorphism with the Risk of End-Stage Renal Disease. Renal Failure, 35, 1295-1301. http://dx.doi.org/10.3109/0886022X.2013.820663

[25] Hallan, S.I., Coresh, J., Astor, B.C., Asberg, A., Powe, N.R., Romundstad, S., et al. (2006) International Comparison of the Relationship of Chronic Kidney Disease Prevalence and ESRD Risk. Journal of the American Society of Nephrology, 17, 2275-2284. http://dx.doi.org/10.1681/ASN.2005121273

[26] Fields, L.E., Burt, V.L., Cutler, J.A., Hughes, J., Roccella, E.J. and Sorlie, P. (2004) The Burden of Adult Hypertension in the United States 1999 to 2000: A Rising Tide. Hypertension, 44, 398-404. http://dx.doi.org/10.1161/01.HYP.0000142248.54761.56

[27] Rao, G.N., Corson, M.A. and Berk, B.C. (1991) Uric Acid Stimulates Vascular Smooth Muscle Cell Proliferation by Increasing Platelet-Derived Growth Factor A-Chain Expression. Journal of Biological Chemistry, 266, 8604-8608.

[28] Susic, D. and Frohlich, E.D. (2011) Hypertensive Cardiovascular and Renal Disease and Target Organ Damage: Lessons from Animal Models. CardioRenal Medicine, 1, 139-146. http://dx.doi.org/10.1159/000329334

[29] Scialla, J.J., Appel, L.J., Astor, B.C., Miller 3rd, E.R., Beddhu, S., Woodward, M., et al. (2012) Net Endogenous Acid Production Is Associated with a Faster Decline in GFR in African Americans. Kidney Internatinal, 82, 106-112. 
http://dx.doi.org/10.1038/ki.2012.82

[30] Luyckx, V.A., Bertram, J.F., Brenner, B.M., Fall, C., Hoy, W.E., Ozanne, S.E., et al. (2013) Effect of Fetal and Child Health on Kidney Development and Long-Term Risk of Hypertension and Kidney Disease. The Lancet, 382, 273-283. http://dx.doi.org/10.1016/S0140-6736(13)60311-6

[31] Lüscher, T.F. and Barton, M. (2000) Endothelins and Endothelin Receptor Antagonists: Therapeutic Considerations for a Novel Class of Cardiovascular Drugs. Circulation, 102, 2434-2440. http://dx.doi.org/10.1161/01.CIR.102.19.2434

[32] Brown, M.J., Coltart, J., Gunewardena, K., Ritter, J.M., Auton, T.R. and Glover, J.F. (2004) Randomized DoubleBlind Placebo-Controlled Study of an Angiotensin Immunotherapeutic Vaccine (PMD3117) in Hypertensive Subjects. Clinical Science (Lond), 107, 167-173. http://dx.doi.org/10.1042/CS20030381

[33] Tissot, A.C., Maurer, P., Nussberger, J., Sabat, R., Pfister, T., Ignatenko, S., et al. (2008) Effect of Immunisation against Angiotensin II with CYT006-AngQb on Ambulatory Blood Pressure: A Double-Blind, Randomised, PlaceboControlled Phase IIa Study. The Lancet, 371, 821-827. http://dx.doi.org/10.1016/S0140-6736(08)60381-5

[34] Navaneethan, S.D., Lohmeier, T.E. and Bisognano, J.D. (2009) Baroreflex Stimulation: A Novel Treatment Option for Resistant Hypertension. Journal of the American Society of Hypertension, 3, 69-74.

[35] Schlaich, M.P., Schmieder, R.E., Bakris, G., Blankestijn, P.J., Böhm, M., Campese, V.M., et al. (2013) International Expert Consensus Statement: Percutaneous Transluminal Renal Denervation for the Treatment of Resistant Hypertension. Journal of the American College of Cardiology, 62, 2031-2045. http://dx.doi.org/10.1016/j.jacc.2013.08.1616

[36] Bhatt, D.L., Kandzari, D.E., O’Neill WW, D’Agostino, R., Flack, J.M., Katzen, B.T., et al. (2014) A Controlled Trial of Renal Denervation for Resistant Hypertension. The New England Journal of Medicine, 370, 1393-1401. http://dx.doi.org/10.1056/NEJMoa1402670

[37] Hicks, L.S., Sequist, T.D., Ayanian, J.Z., Shaykevich, S., Fairchild, D.G., Orav, E.J., et al. (2008) Impact of Computerized Decision Support on Blood Pressure Management and Control: A Randomized Controlled Trial. Journal of General Internal Medicine, 23, 429-441. http://dx.doi.org/10.1007/s11606-007-0403-1 